\section{Supercomputing for the birds}

A database that records the millions of sightings routinely made by dedicated birdwatchers around the globe has just been given some powerful help to analyse its data $-100,000$ hours on the US National Science Foundation's (NSF's) TeraGrid supercomputing network.

The eBird project, run by Steve Kelling, director of information science at the Cornell Laboratory of Ornithology in New York, together with the New York-based National Audubon Society, has been growing steadily since its launch in 2002. More than 48 million observations have been entered, 10 million of them in 2010 alone. According to Kelling, "the challenge now is to try to do something meaningful with all these data".

By performing intensive data analysis using the supercomputer network, Kelling and his colleagues hope to turn the scattered observations of each bird species into a predictive model of its movements.

The eBird team will start by combining the bird sightings with remote-sensing information from sources such as NASA's Terra and Aqua satellites, which can, for example, reveal when different places on Earth are 'greening up' in the spring - a seasonal phenomenon that can be strongly correlated with bird movements. The computers will then 'learn' what kind of land cover, what timing pattern of greening and what human densities best predict bird presence.

With TeraGrid, the Cornell lab plans to marry such models to scenarios for climate change from the Intergovernmental Panel on Climate Change, with the aim of anticipating migratory changes - and perhaps extinctions - for hundreds of species.

These ecological problems are a new frontier for supercomputing, says John Cobb, a principal investigator for TeraGrid at the Oak Ridge National Laboratory in Tennessee and a co-investigator for DataONE, a project sponsored by the NSF to gather and harmonize ecological and environmental data sets. With vast amounts of computing power comes the opportunity to turn the work of many amateur birders into a nuanced portrait of how species migrate. "It is a wonderful story about how they have used all those people who are enthusiastic about birdwatching and made a scientifically significant data set," says Cobb.

Emma Marris

See go.nature.com/dt9WaQ for a longer version of this story and an animation of bird migration.

Correction

The News Feature 'Inside the hothouses of industry' (Nature 466, 548-551; 2010) contained an incorrect statement about Monsanto's partnership with the African Agricultural Technology Foundation (AATF). Monsanto has granted the AATF a perpetual royalty-free licence to its technology for developing droughtresistant crops. Should subsistence farmers become profitable in future, Monsanto could sell to them seeds displaying other traits. 